\title{
Incidencia y factores de riesgo de retinopatía del prematuro en el Hospital Regional Lic. Adolfo López Mateos, ISSSTE
}

\section{Incidence and risk factors of retinopathy of prematurity in the Lic. Adolfo López Mateos Regional Hospital, ISSSTE}

\author{
Dora A. Ochoa-Araujo ${ }^{1 *}$, Vanesa Flores-Peredo ${ }^{1}$, Jessica T. Romero-Martínez ${ }^{1}$, \\ Nancy L. Martínez-Rodríguez ${ }^{2}$ y Oscar Medina-Contreras ${ }^{3 *}$
}

${ }^{1}$ Servicio de Retina, Hospital Regional Lic. Adolfo López Mateos, Instituto de Seguridad de Seguridad y Servicios Sociales de los Trabajadores del Estado; '2Departamento de Investigación en Salud Comunitaria, Hospital Infantil de México Federico Gómez; ${ }^{3}$ Laboratorio de Investigación en Inmunología y Proteómica, Hospital Infantil de México Federico Gómez. Ciudad de México, México

\section{Resumen}

Objetivo: Determinar la incidencia de retinopatía del prematuro (ROP) y analizar los principales factores de riesgo en la población de recién nacidos prematuros del Hospital Lic. Adolfo López Mateos. Método: Se realizó un estudio prospectivo en el que se incluyeron prematuros con edad $\leq 34$ semanas de gestación (SDG), con peso $\leq 2,000 \mathrm{~g}$, durante el periodo de septiembre de 2017 a julio de 2018. Se analizaron factores de riesgo como edad gestacional, peso al nacer, oxigenoterapia, sepsis, transfusión sanguínea, uso de surfactante, uso de esteroide posnatal, síndrome de distrés respiratorio, gestación múltiple, displasia broncopulmonar, persistencia del conducto arterioso, hemorragia intraventricular, episodios de apnea. Se realizó un análisis estadístico para variables continuas y variables categóricas, regresión logística univariada y multivariada. Resultados: Se estudiaron 91 pacientes prematuros, y se observaron 27 casos de ROP (29.6\%). La incidencia de ROP fue mayor en pacientes que pesaban < 1,300 g o < 29 SDG. Se encontró asociación significativa de riesgo para sepsis (OR: 8.48; IC 95\%: 3.07-23.44), SDG $\leq 29$ (OR: 16.3; IC 95\%: 4.06-65.34) y peso al nacer < 1,000 g (OR: 11.25; IC 95\%: 2.89-43.8). Conclusiones: Este estudio demuestra que los principales factores de riesgo para el desarrollo de ROP son sepsis, edad gestacional y peso al nacimiento. La edad gestacional < 29 SDG y el peso al nacer < 1,000 g son particularmente importantes. En un modelo de regresión logística multivariada, la sepsis y el tiempo de gestación fueron los principales factores asociados al desarrollo de ROP. Es importante evaluar a todos los recién nacidos pretérmino de $\leq 34$ SDG y/o $<1,750 \mathrm{~g}$ de peso al nacimiento, y debe prestarse mayor atención a los más pequeños.

Palabras clave: Retinopatía del prematuro. Incidencia. Factores de riesgo.

\footnotetext{
Correspondencia:

*Dora A. Ochoa-Araujo

Fundación H. Nuestra Señora de la Luz

Ezequiel Montes, 135

Col. Tabacalera, Del. Cuauhtemoc

C.P. 06030, Ciudad de México, México

E-mail: alineochoaa@gmail.com $\quad$ Fecha de recepción: 05-04-2019

*Oscar Medina-Contreras $\quad$ Fecha de aceptación: 30-07-2019

E-mail: omedina@ himfg.edu.mx

DOI: 10.24875/RMO.M19000090

Disponible en internet: 01-11-2019 Rev Mex Oftalmol. 2019;93(6):288-294

www.rmo.com.mx 0187-4519/๑ 2019 Sociedad Mexicana de Oftalmología. Publicado por Permanyer México. Este es un artículo Open Access bajo la licencia CC BY-NC-ND (http://creativecommons.org/licenses/by-nc-nd/4.0/).
} 


\section{Abstract}

Objective: To determine the incidence of retinopathy of prematurity (ROP), and to analyze the main risk factors in premature newborns of the Hospital Lic. Adolfo López Mateos. Methods: We performed a prospective study with preterm infants with < 34 weeks of gestational age and a weight < 2000 g, during the period from September 2017 to July 2018. We analyzed risk factors such as gestational age, birth weight, treatment with oxygen, sepsis, blood transfusion, surfactant use, postnatal steroid use, respiratory distress syndrome, multiple pregnancy, bronchopulmonary dysplasia, patent ductus arteriosus, intraventricular hemorrhage, and apnea episodes. We analyzed continuous and categorical variables, performed a univariate logistic regression, and calculated a multivariate logistic regression model. Results: Ninety-one premature patients were studied from which 27 had ROP (29.6\%). ROP incidence was greater in patients weighing $<1300 \mathrm{~g}$ or with a gestational age $<29$ weeks. We found a significant risk association with sepsis (Odds ratio [OR] 8.48, 95\% confident interval [Cl] 3.07-23.44), gestational age $\leq 29$ weeks (16.3,95\% Cl 4.06-65.34), and birth weight < $1000 \mathrm{~g}$ (OR 11.25, 95\% Cl 2.89-43.8). Conclusions: This study demonstrates that the main risk factors for ROP are sepsis, gestational age, and birth weight. A gestational age < 29 weeks and a birth weight < $1000 \mathrm{~g}$ are particularly important. In a multivariate logistic regression model, sepsis and gestational age were the main factors associated with ROP. It is important to evaluate all newborns with a gestational age $\leq 34$ weeks and/or weighing < $1750 \mathrm{~g}$, with particular emphasis to smaller newborns.

Key words: Retinopathy of prematurity. Incidence. Risk factors.

\section{Introducción}

La retinopatía del prematuro $(\mathrm{ROP})$ es una enfermedad retiniana multifactorial en la que existe una interrupción de la vasculogénesis normal y formación de neovasos, que proliferan de manera desordenada y provocan neovascularización, hemorragia vítrea y modificación de la matriz vítrea con aumento de fibroblastos, los cuales se contraen y provocan desprendimiento de retina'.

Esta patología suele asociarse con el nacimiento prematuro, pero el riesgo de que ocurra es una consecuencia de diversos factores interactivos. Sin lugar a dudas, la edad gestacional precoz $(\leq 30$ semanas de gestación [SDG]) y el bajo peso al nacer $(\leq 1,500 \mathrm{~g})$ son los factores de riesgo más importantes en el desarrollo de ROP, pero además de estos existen otros factores de riesgo con impacto significativo ${ }^{2,3}$.

De acuerdo a la Organización Mundial de la Salud, la ROP es la segunda causa más frecuente de ceguera en niños en América Latina ${ }^{4}$.

Globalmente, la ROP sigue siendo la principal amenaza a la visión de los neonatos prematuros. En 2010, se estimó que 184,700 bebés prematuros desarrollaron cualquier etapa de ROP, 20,000 de los cuales quedaron ciegos o con deficiencia visual severa y otros 12,300 desarrollaron deterioro visual leve 0 moderado ${ }^{5}$. Los estudios sugieren que la ROP es una causa cada vez más importante de ceguera evitable en China, sudeste y Asia del sur, América Latina y este de Europa ${ }^{6-8}$.

En EE.UU., la incidencia de ROP aumentó del 14.70\% en 2000 al $19.88 \%$ en 2012 , y las variables que mejor predecían la ROP fueron el sexo femenino, el peso al nacer y la edad gestacional. La frecuencia de ROP fue del $2.40 \%$ en recién nacidos de más de $2,500 \mathrm{~g}$ y del $30.22 \%$ en recién nacidos con un peso al nacer entre 750 y $999 \mathrm{~g}^{9}$.

A pesar de que la prevalencia real de ROP sigue siendo desconocida, en México se han realizado estudios previos para la identificación de ROP en nuestro país. En el Centro Médico Nacional 20 de Noviembre se realizó un estudio prospectivo para determinar la prevalencia de ROP entre 1991 y 2004, en el cual se incluyeron 170 prematuros con peso $<1,500 \mathrm{~g}$ al nacimiento y edad gestacional de $<35$ SDG. Se reportaron 46 lactantes en las etapas I-III de ROP, con una prevalencia del $10.61 \%$, lo que concluye que la incidencia de ROP es inversamente proporcional con la edad gestacional y el peso al nacer de los productos ${ }^{10}$.

Un estudio realizado en el Hospital Dr. Manuel Gea González de la Secretaría de Salud reportó una incidencia del $23 \%{ }^{11}$, y en el Servicio de Neonatología del Hospital Infantil del Estado de Sonora se reportó una incidencia de ROP del $58.1 \%$ en el año $2015^{2}$. En 2012, el Departamento de Pediatría del Instituto Nacional de Perinatología notificó de 139 niños examinados en la Unidad de Cuidados Intensivos, el $24.4 \%$ de los cuales fueron identificados con ROP: $79 \%$ retinopatía grado I, $18 \%$ grado II y un niño con grado III. Las zonas comprometidas fueron: zona I, $12 \%$; zona II, $79 \%$; y zona III, $9 \%$. Los factores de riesgo asociados fueron eclampsia, edad gestacional, gestación múltiple, días de estancia hospitalaria y ventilación mecánica, hipoxia, terapia con $\mathrm{O}_{2}$, apnea, acidosis e hipercapnia ${ }^{12}$ 
Los principales factores de riesgo para ROP son prematuros con edad gestacional $\leq 32 \mathrm{SDG}$, peso al nacimiento $<1,500 \mathrm{~g}$, oxigenoterapia con ventilación mecánica de más de 48 horas con ventilador mecánico conectado a cánula endotraqueal, ventilación nasofaríngea o presión positiva continua en la vía aérea (CPAP). Otros factores descritos son la displasia broncopulmonar, hemorragia intraventricular, sepsis temprana, sepsis tardía, surfactante, esteroides posnatales, transfusión sanguínea y factores de riesgo maternos, como preeclampsia, eclampsia y diabetes ${ }^{4,12-14}$.

De acuerdo a la primera guía de tamizaje de ROP emitida por la Academia Americana de Pediatría, los pacientes prematuros con edad de gestación $\leq 32$ semanas y peso al nacimiento menor de $1,500 \mathrm{~g}$ tienen riesgo de desarrollar retinopatía umbral y desprendimiento de retina y, por tanto, daño visual irreversible ${ }^{13}$. La Secretaría de Salud en México sugiere tamizaje a la siguiente población: a) todos los recién nacidos pretérmino de $\leq 34$ SDG y/o $<1,750 \mathrm{~g}$ de peso al nacimiento, b) a criterio del médico tratante, los recién nacidos pretérmino > 34 SDG y con peso al nacimiento $\geq 1750 \mathrm{~g}$ que hayan recibido oxígeno suplementario, y c) a criterio del médico tratante, los recién nacidos pretérmino que tengan factores de riesgo $0^{15}$; y debe prestarse mayor atención a los más pequeños.

Los recién nacidos prematuros tienen mayor riesgo de desarrollar secuelas en el desarrollo neuromotor, sensorial, cognitivo, así como diversas complicaciones visuales como errores refractivos, ambliopía y estrabismo. Por ello, la identificación temprana y el tratamiento oportuno de prematuros en riesgo es fundamental para evitar el desarrollo de ceguera ${ }^{12}$.

\section{Objetivo}

El objetivo principal del presente trabajo fue reportar la incidencia y factores de riesgo asociados a ROP en el Hospital Regional Lic. Adolfo López Mateos, del Instituto de Seguridad de Seguridad y Servicios Sociales de los Trabajadores del Estado.

\section{Métodos}

Se realizó un estudio prospectivo, descriptivo, durante el periodo de septiembre de 2017 a julio de 2018, en el Hospital Regional. Lic. Adolfo López Mateos. El protocolo fue aprobado por los comités de investigación y ética en investigación del Hospital Regional Lic. Adolfo López Mateos (109.2018 Incidencia de retinopatía del prematuro en un hospital de tercer nivel de atención de la Ciudad de México). Se analizaron prematuros con edad $<34$ SDG y peso $<2,000 \mathrm{~g}$, previo consentimiento informado de los padres.

Los criterios de no inclusión fueron pacientes graves cuyas condiciones sistémicas no permitían su exploración. Fueron eliminados los pacientes que no fueron llevados a cita de control en consulta externa y los que fallecieron.

La captación de pacientes se llevó a cabo mediante exploración de fondo de ojo de los pacientes prematuros, y se dividieron en presencia o ausencia de ROP, y en caso de presentarla, por la estratificación de esta. Se analizaron factores de riesgo como edad gestacional, peso al nacer, oxigenoterapia, sepsis, transfusión sanguínea, uso de surfactante, uso de esteroide posnatal, síndrome de distrés respiratorio, gestación múltiple, displasia broncopulmonar, persistencia del conducto arterioso, hemorragia intraventricular, episodios de apnea, y factores de riesgo maternos como preeclampsia, diabetes gestacional, síndrome de HELLP (Hemolytic anemia, Elevated Liver enzyme, Low Platelet count), y eclampsia.

El examen retiniano se efectuó tras la aplicación de gotas oftálmicas con tropicamida y fenilefrina diluida al $50 \%$, una gota en cada ojo cada 10 minutos en tres ocasiones. Se aplicó anestesia tópica (tetracaína) y se valoró mediante oftalmoscopia binocular indirecta y lupa de 28 dioptrías, utilizando un blefarostato pediátrico o separadores tipo desmarres con indentación de la periferia retiniana. Al concluir la exploración se aplicaron gotas de antibiótico tópico (tobramicina). Los pacientes fueron examinados a las 4 semanas del nacimiento y los más pequeños al cumplir 31 semanas de edad gestacional corregida. Las evaluaciones se realizaron cada 10 2 semanas en la unidad de cuidados intensivos neonatal o por consulta externa, dependiendo de la condición clínica del paciente, hasta completar la vasculatura retiniana y encontrar remisión de la retinopatía.

Los datos fueron consignados en una hoja electrónica utilizando el programa Stata, y se realizó su análisis estadístico con las pruebas U-Mann-Whitney para variables continuas y Chi-cuadrada o Fisher para variables categóricas. Se realizó también regresión logística univariada y un modelo de regresión logística multivariada.

\section{Resultados}

En este estudio se incluyeron 91 prematuros con factores de riesgo para ROP (182 ojos). En total se identificaron 27 pacientes con ROP y 64 pacientes sin ROP, lo que resulta en una incidencia de ROP del $29.6 \%$. En el grupo de pacientes sin ROP se observó 
un discreto predominio masculino ( $n=34,53.1 \%$ ), mientras que entre los pacientes con ROP hubo un discreto predominio femenino $(n=15,55.6 \%)$.

$\mathrm{Al}$ analizar las diferentes variables de características clínicas se encontró una presencia significativa de ROP entre los pacientes que presentaron sepsis (70.4\%). Además de la sepsis, el tiempo de gestación y el peso al nacer presentaron diferencias significativas importantes (Tabla 1).

En el grupo de pacientes con ROP se observó una mayor frecuencia de conducto arterioso persistente (37\%), uso de esteroides posnatales (37\%) y uso de ventilación mecánica prolongada (59.3\%). De manera interesante, solamente 4 necesitaron tratamiento antiangiogénico.

Con respecto a factores de riesgo relacionados al embarazo, en el grupo ROP se observó mayor diabetes gestacional $(29.6 \%)$ y mayor frecuencia de gestación múltiple (Tabla 1).

Se realizó una regresión logística univariada para comparar entre los factores de riesgo de los pacientes que presentaron ROP y los del grupo sin ROP. De manera similar a las frecuencias, se identificó la sepsis como el principal factor asociado a ROP (OR: 8.48; IC 95\%: 3.07-23.44; $p<0.001$ ) (Tabla 2).

El tiempo de gestación y el peso al nacer también presentaron un alto factor de riesgo para el desarrollo de ROP. El tiempo de gestación < 29 SDG fue el principal factor de riesgo (OR: 16.3; IC 95\%: 4.0665.34; $p<0.001$ ), seguido de un peso al nacer $<1,000$ g (OR: 11.25; IC 95\%: 2.89-43.8; $p<0.001$ ).

Se utilizó el análisis de regresión logística múltiple para evaluar los principales factores de riesgo para el desarrollo de ROP. El modelo que mejor explica la asociación es el que se encuentra ajustado por sepsis, edad gestacional y peso al nacer (Tabla 3). Este modelo confirmó que la sepsis es el principal factor asociado al desarrollo de ROP, seguido del tiempo de gestación, donde el riesgo de desarrollar ROP es de 5.28 veces más en pacientes con sepsis y de 0.77 veces más por cada semana menos de edad gestacional. De manera interesante, el peso al nacer no contribuye significativamente al riesgo en este modelo.

\section{Discusión}

En este estudio determinamos la incidencia de ROP y sus principales factores de riesgo en la población de recién nacidos prematuros del Hospital Lic. Adolfo López Mateos. Se incluyeron a los prematuros con peso $\leq 2,000 \mathrm{~g}$ y edad gestacional $\leq 34 \mathrm{SDG}$, basándose en las recomendaciones del grupo ROP y de la Secretaría de Salud de México ${ }^{15}$. De la población analizada en este estudio, 30 pacientes (30\% del total) tenían un peso $>1,750 \mathrm{~g}$ con menos de $34 \mathrm{SDG}$. De acuerdo con los criterios para los pacientes con ROP de la Secretaría de Salud y el Grupo ROP, si el paciente se expuso a oxígeno, se considera como riesgo de desarrollar ROP, independientemente del peso. Debido a que el $93 \%$ de nuestros pacientes con peso > 1,750 g recibió oxígeno suplementario, decidimos considerar a estos pacientes con riesgo a pesar de tener un peso superior.

La incidencia de ROP observada en este estudio fue del $29.6 \%$, la cual es similar a la reportada en otros países, incluyendo el $30.4 \%$ en Pakistán ${ }^{16}$, el $29.5 \%$ en Irán ${ }^{17}$ o el $29.2 \%$ en Singapur ${ }^{18}$, aunque mayor a la reportada en EE.UU, que es del $10.45 \%{ }^{19}$. Esto sugiere que no hay una mayor predisposición genética o ambiental a desarrollar ROP en nuestra población, aunque existen factores asociados al nivel de desarrollo que disminuyen este riesgo.

La incidencia de ROP fue mayor en pacientes con pero $<1,300$ g $0<29$ SDG. Se encontró asociación significativa de riesgo para sepsis (OR: 8.48; IC 95\%: 3.07-23.44), SDG $\leq 29$ (OR: 16.3; IC95\%: 4.06-65.34) y peso al nacer < 1,000 g (OR: 11.25; IC 95\%: 2.89-43.8).

En nuestra población identificamos una fuerte asociación con la edad gestacional $(p<0.001)$ y peso al nacer ( $p<0.001$ ), lo cual coincide con lo reportado a nivel mundial. También confirmamos que la incidencia de la ROP es inversamente proporcional con la edad gestacional y el peso al nacer de los productos, es decir, a menor edad gestacional y peso, mayor incidencia de ROP ${ }^{10,13,20-22}$.

Además, observamos una asociación significativa del tiempo total de oxígeno suplementario en pacientes con ROP, el cual fue de 26 días ( $p=0.007$ ), apoyando la asociación que representa como factor de riesgo determinante para la presentación de ROP ${ }^{12}$.

La diabetes gestacional y la persistencia del conducto arterioso reportados previamente con asociación significativa con ROP, en nuestro estudio también tuvieron una asociación significativa $(p=0.028$ y $p=0.024$, respectivamente).

En el uso de ventilación mecánica, la entrada de oxigeno es alta, a pesar de utilizar presiones normales o bajas, por lo que el uso prolongado puede estar correlacionado con una alta incidencia de ROP. También observamos una asociación estadísticamente significativa $(p=0.029)$, a pesar de las medidas de regulación de estos parámetros en la unidad de cuidados intensivos neonatales $2,4,12,14$. 
Rev Mex Oftalmol. 2019;93

Tabla 1. Características clínicas

\begin{tabular}{|c|c|c|c|c|}
\hline Parámetro & Todos $(\mathrm{n}=91)$ & $\operatorname{Sin} \operatorname{ROP}(n=64)$ & $\mathrm{ROP}(\mathrm{n}=27)$ & $\mathbf{p}$ \\
\hline Sepsis & $33(36.3)$ & $14(21.9)$ & $19(70.4)$ & $<0.001$ \\
\hline Persistencia del conducto arterioso & $20(22.0)$ & $10(15.6)$ & $10(37.0)$ & 0.024 \\
\hline Diabetes gestacional & $15(16.5)$ & $7(10.9)$ & $8(29.6)$ & 0.028 \\
\hline Gestación múltiple & $28(30.8)$ & $16(25.0)$ & $12(44.4)$ & 0.066 \\
\hline Ventilación mecánica prolongada & $41(45.1)$ & $25(39.1)$ & $16(59.3)$ & 0.077 \\
\hline Sexo femenino & $45(49.5)$ & $30(46.9)$ & $15(55.6)$ & 0.449 \\
\hline Flujo libre & $47(51.6)$ & $31(48.4)$ & $16(59.3)$ & 0.224 \\
\hline Casco cefálico & $47(51.6)$ & $32(50.0)$ & $15(55.6)$ & 0.535 \\
\hline Puntas nasales & $42(46.2)$ & $27(42.2)$ & $15(55.6)$ & 0.191 \\
\hline CPAP & $22(24.2)$ & $18(28.1)$ & $4(14.8)$ & 0.224 \\
\hline Ruptura prematura de membranas & $26(28.6)$ & $16(25.0)$ & $10(37.0)$ & 0.077 \\
\hline Parto pretérmino & $36(39.6)$ & $25(39.1)$ & $11(40.7)$ & 0.246 \\
\hline Infecciones de vías urinarias & $20(22.0)$ & $16(25.0)$ & $4(14.8)$ & 0.881 \\
\hline Placenta previa & $4(4.4)$ & $4(6.3)$ & $0(0.0)$ & 0.355 \\
\hline Síndrome de HELLP & $2(2.2)$ & $1(1.6)$ & $1(3.7)$ & 0.184 \\
\hline Infecciones cervicovaginales & $18(19.8)$ & $13(20.3)$ & $5(18.5)$ & 0.525 \\
\hline Preeclampsia & $36(39.6)$ & $26(40.6)$ & $10(37.0)$ & 0.844 \\
\hline Episodios de apnea & $13(14.3)$ & $9(14.1)$ & $4(14.8)$ & 0.749 \\
\hline Uso de surfactante & $26(28.9)$ & $17(26.9)$ & $9(33.3)$ & 0.925 \\
\hline Esteroides posnatales & $23(25.3)$ & $13(20.3)$ & $10(37.0)$ & 0.441 \\
\hline Síndrome de distrés respiratorio & $37(40.7)$ & $23(37.5)$ & $13(48.1)$ & 0.063 \\
\hline Displasia broncopulmonar & 12 (13.2) & $10(15.6)$ & $2(7.4)$ & 0.277 \\
\hline $\begin{array}{l}\text { Gestación (semanas) } \\
\leq 29 \\
29.1-32 \\
32.1-34\end{array}$ & $\begin{array}{l}16(15.6) \\
30(33.0) \\
45(49.4)\end{array}$ & $\begin{array}{c}4(6.25) \\
22(34.4) \\
38(59.4)\end{array}$ & $\begin{array}{l}12(44.4) \\
8(29.6) \\
7(25.9)\end{array}$ & $<0.001$ \\
\hline $\begin{array}{l}\text { Peso al nacer (gramos) } \\
\leq 1,000 \\
1,001-1,300 \\
1,301-2,000\end{array}$ & $\begin{array}{l}13(14.3) \\
18(19.8) \\
60(65.9)\end{array}$ & $\begin{array}{c}4(6.2) \\
10(15.6) \\
50(78.1)\end{array}$ & $\begin{array}{c}9(33.3) \\
8(29.6) \\
10(37.0)\end{array}$ & $<0.001$ \\
\hline Tiempo total de $\mathrm{O}_{2}$ suplementario (días) & $10(3-30)$ & $8(3-24.5)$ & $26(7-35)$ & 0.007 \\
\hline Tiempo de ventilación mecánica (días) & $0(0-7)$ & $0(0-4)$ & $5(0-12)$ & 0.029 \\
\hline Tiempo con puntas nasales (días) & $0(0-3)$ & $0(0-3)$ & $2(0-5)$ & 0.054 \\
\hline Tiempo de flujo libre (días) & $1(0-6)$ & $0(0-6)$ & $2(0-7)$ & 0.423 \\
\hline Tiempo de casco cefálico (días) & $1(0-4)$ & $0.5(0-3)$ & $2(0-10)$ & 0.119 \\
\hline
\end{tabular}

En conjunto, estos parámetros asociados al desarrollo de ROP nos sugieren que nuestra población no tiene mayor predisposición que la observada a nivel mundial.
De manera interesante, de los 91 prematuros estudiados, solamente 4 necesitaron tratamiento antiangiogénico; 2 de estos prematuros tenían 9 factores de riesgo 
Tabla 2. Factores de riesgo para el desarrollo de retinopatía del prematuro

\begin{tabular}{|c|c|c|}
\hline Parámetro & OR (IC 95\%) & p \\
\hline Sepsis & $8.48(3.07-23.44)$ & $<0.001$ \\
\hline $\begin{array}{l}\text { Persistencia del conducto } \\
\text { arterioso }\end{array}$ & $3.18(1.13-8.92)$ & 0.028 \\
\hline Diabetes gestacional & $3.43(1.10-10.71)$ & 0.034 \\
\hline $\begin{array}{l}\text { Gestación (semanas) } \\
\leq 29 \\
29.1-32 \\
32.1-34\end{array}$ & $\begin{array}{c}0.67(0.54-0.83) \\
16.3(4.06-65.34) \\
1.97(0.63-6.18) \\
1.0\end{array}$ & $\begin{array}{c}<0.001 \\
<0.001 \\
0.243\end{array}$ \\
\hline $\begin{array}{l}\text { Peso al nacer (gramos) } \\
\leq 1,000 \\
1,001-1,300 \\
1,301-2,000\end{array}$ & $\begin{array}{c}0.99(0.996-0.999) \\
11.25(2.89-43.8) \\
4.0(1.26-12.64) \\
1.0\end{array}$ & $\begin{array}{c}<0.001 \\
<0.001 \\
0.018 \\
-\end{array}$ \\
\hline $\begin{array}{l}\text { Tiempo total de } \mathrm{O}_{2} \\
\text { suplementario (días) }\end{array}$ & $1.04(1.01-1.06)$ & 0.009 \\
\hline $\begin{array}{l}\text { Tiempo de ventilación } \\
\text { mecánica (días) }\end{array}$ & $1.06(1.01-1.12)$ & 0.014 \\
\hline $\begin{array}{l}\text { Tiempo con puntas } \\
\text { nasales (días) }\end{array}$ & $1.11(1.01-1.22)$ & 0.033 \\
\hline
\end{tabular}

Tabla 3. Evaluación de riesgo para retinopatía del prematuro

\begin{tabular}{|l|c|c|c|}
\hline Modelo & $z$ & OR (IC 95\%) & P \\
\hline Sepsis & 2.88 & $5.28(1.70-16.37)$ & $\mathbf{0 . 0 0 4}$ \\
\hline Gestación (semanas) & -2.07 & $0.77(0.61-0.99)$ & $\mathbf{0 . 0 3 8}$ \\
\hline Peso al nacer (gramos) & -1.50 & $0.99(0.99-1.00)$ & 0.134 \\
\hline
\end{tabular}

(peso extremadamente bajo de 700 y $800 \mathrm{~g}$, respectivamente), y recibieron oxígeno durante más de 45 días y estuvieron 30 días con ventilación mecánica.

Se ha reportado que los periodos de apnea son el factor de riesgo más significativo para el desarrollo de ROP23; sin embargo, en nuestro estudio no encontramos asociación significativa. Esto podría explicarse a la asociación de los periodos de apnea con condiciones patológicas agudas, pero no con las crónicas como ROP24, y a una mejor oxigenación en los prematuros con ROP en la unidad de cuidados intensivos neonatales.

La Academia Americana de Pediatría ha recomendado el uso de esteroides posnatales solamente en recién nacidos con falla en la extubación o con ventilación mecánica prolongada; sin embargo, no observamos un efecto de estos en el riesgo a desarrollar ROP. Esta observación es consistente con diversos estudios, donde no reportan ningún efecto del uso de esteroides posnatales en la incidencia de ROP ${ }^{15,25}$.

Esta evidencia parece sugerir que los periodos de apnea y el uso de esteroides tienen un mejor manejo en nuestra unidad de cuidados intensivos, por lo que no contribuyen al desarrollo de ROP en nuestra población.

Múltiples estudios han reportado el papel de la sepsis neonatal en el desarrollo de ROP ${ }^{21,26}$. En este estudio, la sepsis fue un factor de riesgo con asociación significativa para el desarrollo de ROP $(p<0.001)$ : estuvo presente en el $57.6 \%$ de los pacientes con ROP y se asoció principalmente con el desarrollo de ROP estadio I (33.3\%) y II (24.4\%). Esto se explica por el proceso secundario mediado a través de citocinas y endotoxinas que afectan directamente la angiogénesis retiniana. Este proceso se acompaña frecuentemente de hipotensión, que puede causar deterioro de la perfusión tisular e isquemia retiniana ${ }^{27,28}$.

Los recién nacidos prematuros son particularmente susceptibles a infecciones, debido al bajo peso al nacer y a la edad gestacional. La exposición del recién nacido prematuro a la infección y mediadores inflamatorios, y el consecuente desarrollo de sepsis de inicio temprano, se asocia con un mayor riesgo de desarrollar ROP ${ }^{21,26}$, principalmente en edad gestacional extremadamente baja. En este estudio encontramos una asociación significativa $(p<0.001)$ de la sepsis con el riesgo de desarrollar ROP, con una incidencia relativamente alta (57.6\%). Sin embargo, es posible que en el estadio III también exista una fuerte asociación, que posiblemente no pudimos detectar debido que eran prematuros cuyas graves condiciones sistémicas no permitieron su exploración.

En resumen, en el Hospital Regional Lic. Adolfo López Mateos encontramos que la edad gestacional y el peso al nacer contribuyen de manera significativa con el desarrollo de ROP. Observamos una fuerte asociación de sepsis con el desarrollo de ROP, por lo que este factor de riesgo debe de ser evaluado y tratado con especial atención, ya que es modificable.

A pesar de que la tendencia no es similar en todas las unidades, actualmente los programas de vigilancia y tamizaje permiten la detección temprana, lo cual se ha reflejado con menor incidencia de estadios avanzados de la enfermedad. La disminución en la incidencia de ROP avanzado refleja un mayor conocimiento de los factores de riesgo y su manejo a tiempo, así como la mejora de la atención neonatal en nuestra unidad. 


\section{Conclusiones}

Nuestro estudio demuestra que los principales factores de riesgo para el desarrollo de ROP son sepsis, edad gestacional y peso al nacer. Dentro de estos últimos factores, la edad gestacional $<29$ SDG y peso al nacer $<1,000 \mathrm{~g}$ tuvieron el mayor grado de asociación con el riesgo a desarrollar ROP.

El modelo análisis de regresión logística multivariada sitúa la sepsis como el principal factor asociado al desarrollo de ROP, seguido del tiempo de gestación. De manera interesante, el peso al nacer no contribuye significativamente al riesgo en este modelo.

\section{Conflicto de intereses}

Los autores declaran no tener conflicto de intereses.

\section{Responsabilidades éticas}

Protección de personas y animales. Los autores declaran que los procedimientos seguidos se conformaron a las normas éticas del comité de experimentación humana responsable y de acuerdo con la Asociación Médica Mundial y la Declaración de Helsinki.

Confidencialidad de los datos. Los autores declaran que han seguido los protocolos de su centro de trabajo sobre la publicación de datos de pacientes.

Derecho a la privacidad y consentimiento informado. Los autores han obtenido el consentimiento informado de los pacientes y/o sujetos referidos en el artículo. Este documento obra en poder del autor de correspondencia.

\section{Bibliografía}

1. Alajbegovic-Halimic J, Zvizdic D, Alimanovic-Halilovic E, Dodik I, Duvnjak S. Risk factors for retinopathy of prematurity in premature born children. Med Arch. 2015;69(6):409-13.

2. Martínez-Ruiz J, Martínez-Carballo E, Ramírez-Rodríguez C. Incidencia de retinopatía del prematuro en el servicio de neonatología del Hospital Infantil del Estado de Sonora. Bol Clin Hosp Infant Edo Son. 2015;32(2):82-6.

3. Mitsiakos G, Papageorgiou A. Incidence and factors predisposing to retinopathy of prematurity in inborn infants less than 32 weeks of gestation. Hippokratia. 2016;20(2):121-6.

4. Ramírez-Ortíz MA, Villa-Guillén $M$, Villanueva-García $D$ Murguía-de Sierra T, Saucedo-Castillo A, Etulain-González A. Criterios de tamizaje en el examen ocular de prematuros mexicanos con riesgo de desarrollo de ceguera irreversible por retinopatía de la prematurez. Bol Med Hosp Infant Mex. 2008;65:179-85.

5. Blencowe H, Lawn JE, Vazquez T, Fielder A, Gilbert C. Preterm-associated visual impairment and estimates of retinopathy of prematurity at regional and global levels for 2010. Pediatr Res. 2013;74 Suppl 1:35-49.
6. Gilbert C, Rahi J, Eckstein M, O'Sullivan J, Foster A. Retinopathy of prematurity in middle-income countries. The Lancet. 1997;350(9070): $12-4$.

7. Limburg H, Gilbert C, Hon DN, Dung NC, Hoang TH. Prevalence and causes of blindness in children in Vietnam. Ophthalmology. 2012;119(2): 355-61.

8. Zepeda-Romero LC, Barrera-de-Leon JC, Camacho-Choza C, Gonzalez Bernal C, Camarena-Garcia E, Diaz-Alatorre C, et al. Retinopathy of prematurity as a major cause of severe visual impairment and blindness in children in schools for the blind in Guadalajara city, Mexico. $\mathrm{Br} J$ Ophthalmol. 2011;95(11):1502-5

9. Ludwig CA, Chen TA, Hernandez-Boussard T, Moshfeghi AA, Moshfeghi DM. The epidemiology of retinopathy of prematurity in the United States. Ophthalmic Surg Lasers Imaging Retina. 2017;48(7):553-62.

10. Orozco-Gomez LP, Ruiz-Morfin I, Lambarry-Arroyo A, Morales-Cruz MV. Prevalencia de retinopatia del prematuro. 12 años de deteccion en el Centro Medico 20 de Noviembre. Cir Cir. 2006;74(1):3-9.

11. González-Urquidi O, Fuente-Torres M. Incidencia de retinopatía del prematuro en el hospital Dr. Manuel Gea González. Rev Mex Oftalmol. 2004; 78(1):1-4.

12. Martinez-Cruz CF, Salgado-Valladares M, Poblano A, Trinidad-Perez MC. Risk factors associated with retinopathy of prematurity and visual alterations in infants with extremely low birth weight. Rev Invest Clin. 2012;64(2):136-43.

13. American Academy of Pediatrics. Section on O. Screening examination of premature infants for retinopathy of prematurity. Pediatrics. 2001; 108(3):809-11.

14. Zamorano Jimenez CA, Salgado Valladares M, Velasquez Valassi B. Risk factors associated to retinopathy of prematurity. Gac Med Mex. 2012;148(1):19-25.

15. CENETEC. Detección, diagnóstico y tratamiento de la retinopatía del prematuro en el segundo y tercer nivel de atención. Secretaría de Salud. 2015:IMSS-281-10:3-15.

16. Taqui AM, Syed R, Chaudhry TA, Ahmad K, Salat MS. Retinopathy of prematurity: frequency and risk factors in a tertiary care hospital in Karachi, Pakistan. J Pak Med Assoc. 2008;58(4):186-90.

17. Karkhaneh R, Mousavi SZ, Riazi-Esfahani M, Ebrahimzadeh SA, Roohipoor R, Kadivar M, et al. Incidence and risk factors of retinopathy of prematurity in a tertiary eye hospital in Tehran. $\mathrm{Br} \mathrm{J}$ Ophthalmol. 2008; 92(11):1446-9

18. Shah VA, Yeo CL, Ling YLF, Ho LY. Incidence, risk factors of retinopathy of prematurity among very low birth weight infants in Singapore. Ann Acad Med Singap. 2005;34(2):169-78.

19. Lad EM, Nguyen TC, Morton JM, Moshfeghi DM. Retinopathy of prematurity in the United States. Br J Ophthalmol. 2008;92(3):320-5.

20. Terry TL. Extreme prematurity and fibroblastic overgrowth of persisten vascular sheath behind each crystalline lens: I. preliminary report. Am J Ophthalmol. 2018;192:xxviii.

21. Tolsma KW, Allred EN, Chen ML, Duker J, Leviton A, Dammann O. Neonatal bacteremia and retinopathy of prematurity: the ELGAN study. Arch Ophthalmol. 2011;129(12):1555-63.

22. Yannuzzi L, Guyet D, Green W. Retinopathy of prematurity. The Retina Atlas. 1996:470-9.

23. Kim TI, Sohn J, Pi SY, Yoon YH. Postnatal risk factors of retinopathy of prematurity. Paediatr Perinat Epidemiol. 2004;18(2):130-4.

24. Fairchild K, Mohr M, Paget-Brown A, Tabacaru C, Lake D, Delos J, et al. Clinical associations of immature breathing in preterm infants: part 1-central apnea. Pediatr Res. 2016;80(1):21-7.

25. Banks BA, Cnaan A, Morgan MA, Parer JT, Merrill JD, Ballard PL, et al. Multiple courses of antenatal corticosteroids and outcome of premature neonates. North American Thyrotropin-Releasing Hormone Study Group. Am J Obstet Gynecol. 1999;181(3):709-17.

26. Stoll BJ, Hansen NI, Adams-Chapman I, Fanaroff AA, Hintz SR, Vohr B, et al. Neurodevelopmental and growth impairment among extremely low-birth-weight infants with neonatal infection. JAMA. 2004;292(19): 2357-65.

27. Lee J, Dammann O. Perinatal infection, inflammation, and retinopathy of prematurity. Semin Fetal Neonatal Med. 2012;17(1):26-9.

28. Hong HK, Lee HJ, Ko JH, Park JH, Park JY, Choi CW, et al. Neonatal systemic inflammation in rats alters retinal vessel development and simulates pathologic features of retinopathy of prematurity. J Neuroinflammation. 2014;11:87. 